\title{
Subtotal colectomy for ulcerative colitis: lessons learned from a tertiary center
}

Nicolas C. BUCHS ${ }^{1}$ MD PD, Alexander L. A. BLOEMENDAAL ${ }^{1}$ MD PhD, Christopher P.J. WOOD ${ }^{1}$ MEd, Simon TRAVIS ${ }^{2}$ DPhil FRCP, Neil J. MORTENSEN ${ }^{1}$ MD, Richard J. GUY ${ }^{1}$ MD, Bruce D. GEORGE ${ }^{1}$ MD.

${ }^{1}$ Department of Colorectal Surgery, Churchill Hospital, Oxford University Hospitals, Oxford, UK

${ }^{2}$ Translational Gastroenterology Unit, Nuffield Department of Medicine, University of Oxford, Oxford, UK

\section{Correspondence to:}

Nicolas C. BUCHS, MD

Department of Colorectal Surgery

Churchill Hospital, Oxford University Hospitals

Old Road, OX3 7LE, Oxford, UK

Nicolas.c.buchs@hcuge.ch

RUNNING TITLE: Subtotal colectomy for ulcerative colitis.

WORD COUNT: 3020

ABSTRACT WORD COUNT: 255

FIGURE: 1 TABLES: 4

Disclosures: The authors have no conflicting financial issues for disclosure.

KEYS WORDS: ulcerative colitis, colectomy, laparoscopy, complications, outcomes, ileoanal pouch.

Presented as a poster at the $11^{\text {th }}$ ECCO meeting, Amsterdam, March 16-19 2016. 


\section{ABSTRACT}

Background: Subtotal colectomy (STC) is a well-established treatment for complicated and refractory ulcerative colitis (UC). A laparoscopic approach offers potentially improved outcomes. The aim of this study is to report our experience with STC for UC in a single large center.

Material and Methods: From January 2007 to May 2015, all consecutive patients undergoing STC for UC were retrospectively analyzed from a prospectively-managed database. Patients with known Crohn's disease or those undergoing one-stage procedures were excluded. Demographics, perioperative outcomes and second-stage procedures were analyzed.

Results: During the study period, 151 STC were performed for UC (100 emergency (66\%) and 51 elective (34\%)). Acute severe colitis refractory to therapy were the most common indications $(61.6 \%)$. Overall, 117 laparoscopic (77.5\%) and 34 open STC were performed, with a conversion rate of $14.5 \%$. Mortality and morbidity rates were $0.7 \%$ and $38.4 \%$, respectively. Whilst operative time was shorter for open STC (by 75 minutes; $p=0.001$ ), there were fewer complications (31.6\% vs. 61.9\%; $\mathrm{p}=0.002$ ) and a shorter hospital stay (by 6.9 days; p=0.0002) following laparoscopic STC. Fewer complications and shorter hospital stay were also observed after elective STC. Patients undergoing laparoscopic STC were more likely to undergo a restorative second-stage procedure than those having open STC (75.3\% vs. $50 \% ; \mathrm{p}=0.03)$.

Conclusions: Laparoscopic STC for UC is feasible and safe, even in the emergency situation. A laparoscopic approach may offer advantages in terms of lower morbidity and reduced length of stay. Elective resection may offer similar advantages and is best performed whenever possible. 


\section{INTRODUCTION}

Current medical therapies for ulcerative colitis (UC) are inadequate to achieve remission in all patients ${ }^{1}$. Despite developments in medical treatment, many patients with UC require subtotal colectomy (STC). This represents $40 \%$ of patients with severe acute colitis ${ }^{2}$. The overall colectomy rate for patients with UC varies, depending on the center and population ${ }^{3}$, but in Oxford the rate is $12 \%^{4}$ and patients fall into two groups. For the $75 \%$ of patients who never have a hospital admission for acute severe colitis the rate is 3\%, but for the other $25 \%$ it is $40 \%$. In cases of long- standing UC, patients must often choose between surgery and an escalation of medical therapy, involving steroids and immunosuppressant therapy ${ }^{1}$.

Standard surgical indications have therefore been extended to treat not only complications of UC (perforation, megacolon, haemorrhage, malignant transformation), but also colitis that is refractory to medical therapy. Such patients are usually those hospitalized with acute severe colitis, although in some centers (especially in the USA) with a large tertiary referral practice, there are more elective colectomies on outpatients with treatment-refractory colitis, simply because there are still well enough to travel for surgery at a specialist center. In acute severe colitis, the option of choice remains subtotal colectomy (STC) with end ileostomy. The rectal stump is preserved, and any restorative procedure (such as ileal pouch-anal anastomosis) is delayed until steroids have been stopped and the patient's general condition has improved.

Minimally invasive surgery is well established in colorectal surgery, including surgery for inflammatory bowel disease ${ }^{5,6}$. Feasibility and safety of laparoscopic STC for UC have been recognized in several studies ${ }^{7-10}$. Beyond short-term advantages, such as reduction of surgical site infection and shorter hospital stay ${ }^{11}$, the laparoscopic approach may facilitate subsequent restorative surgery ${ }^{12}$. 
The aim of this study is to report our experience with STC for UC in a single institution with a large secondary care service for $640 ’ 000$ population and a small proportion $(<20 \%)$ of tertiary referrals.

\section{MATERIALS AND METHODS}

From January 2007 to March 2015, all consecutive patients who underwent STC for UC were retrospectively reviewed. All procedures were performed by colorectal consultants, or by senior trainees under their supervision.

Both laparoscopic and open STC were included. Cases known as Crohn's disease were excluded from the analysis. Patients undergoing total proctocolectomy, with or without ileal pouch anal anastomosis (IPAA), were also excluded.

The decision to proceed to emergency STC was always made collaboratively between surgeons and gastroenterologists; less urgent decisions were made by a multidisciplinary team (MDT). Severity of UC was based on clinical, endoscopic, histological and radiological investigations. The definition of acute severe colitis was based on Truelove and Witts' criteria $^{13}$. Patients' demographics, perioperative outcomes, and subsequent second stage procedures were analyzed. Elective STC was defined as a planned operation performed on the day of planned admission ${ }^{9}$. Emergency STC was defined as an operation performed for a hospitalized patient after failure of optimal medical treatment or for complications. Operative time was defined as the time between the first incision to placement of the last suture.

Complications were classified according to the Clavien-Dindo system ${ }^{14}$.

Operative technique 
The selection of a particular operative approach (open or laparoscopic) was left to the discretion of the individual operating surgeon and anaesthetist. Patients were placed in a modified Lloyd Davies position. Urinary catheterization and rectal washout were routinely performed.

Prophylatic antimicrobial therapy was given just before the initial skin incision.

For patients undergoing laparoscopic STC, an umbilical $10 \mathrm{~mm}$ port was used for the camera, and four $5 \mathrm{~mm}$ ports were added in each of the four quadrants. The dissection started at the rectosigmoid junction and an anti-clockwise division of the colonic mesentery was performed. The superior rectal artery was preserved. The rectosigmoid junction was transected using a laparoscopic linear stapler (the $5 \mathrm{~mm}$ right iliac fossa port being changed for a $10 \mathrm{~mm}$ at the proposed ileostomy site). The colectomy specimen was removed via the ileostomy site, a small Pfannenstiel incision or a short vertical trans-umbilical incision. A wound protector was used. A standard end ileostomy was then matured. A 30 Foley rectal catheter was routinely inserted to allow drainage of the rectal stump and removed after 2-3 days.

For patients having an open STC, a midline incision was performed. A wound protector was routinely used. Dissection was started either at the caecum or at the rectosigmoid junction, which was divided using a linear stapler. As with the laparoscopic STC cases a rectal catheter was also routinely inserted.

Conversion was defined as the inability to complete the mobilization of the colon laparoscopically, resulting in a larger incision than would otherwise have been required for the removal of the resected specimen. The converted patients were analyzed as part of the laparoscopic group (intention to treat).

Statistical analysis 
The results of parametric and nonparametric data were expressed as mean \pm standard deviation (SD) and median (range), respectively. GraphPad Software (GraphPad, La Jolla CA) was used for all statistical analyses. Confidence intervals were set at $95 \%$. A 2 -sided $P$ value of $\leq 0.05$ was considered statistically significant. Comparisons between both groups were determined using Fisher's exact test for discrete variables and Student's t-test for continuous variables.

\section{RESULTS}

\section{Patients' characteristics}

During the study period, 151 patients underwent STC for UC. Patients' demographics are summarized in Table 1. Most patients were male (61.6\%), with a mean age of $43.4+/-18.1$ years (range: 14.2-90.8). The mean ASA (American Society of Anaesthesiologists) score was $2.1+/-$ 0.6, ranging from 1 to 4 . Patients were generally overweight, with a mean BMI (Body Mass Index) of $28.9+/-7.2 \mathrm{~kg} / \mathrm{m}^{2}$ (range: $20-44$ ). Acute severe colitis refractory to therapy was the most frequent indications for STC, accounting for $62 \%$ of all cases (93/151). Patients with persistently active UC in spite of optimal medical therapy (steroid-dependent) represented $9 \%$ of the overall series. Megacolon accounted for 7\% and dysplasia (or malignancy) $4 \%$ of the cases, respectively.

During the study period, 100 emergency $(66.2 \%)$ and 51 elective $(33.8 \%)$ STC were performed. Patients' demographics were similar for both groups (Table 1), except the ASA score, which was higher in the emergency group $(+0.3 ; \mathrm{p}=0.007)$. The indications differed between the two groups: there were more patients with acute severe colitis and megacolon in the emergency group $(\mathrm{p}<0.05)$, whereas patients with steroid-dependent UC were more likely to undergo 
elective STC. The median preoperative length of stay for patients undergoing emergency STC was 2 days (range: $0-13$ ).

Over time, there was a change in operative approach (Figure 1). In 2007 most STC were performed via an open approach, there was a subsequent trend towards laparoscopy, and since 2009 most STC have been performed by a minimally invasive approach. Overall, 117 laparoscopic (77.5\%) and 34 open (22.5\%) STC were performed. There were differences in terms of demographics (Table 1). In the open group, there were comparably more male patients $(+20 \% ; \mathrm{p}=0.05)$, the population was older $(+9.1$ years; $\mathrm{p}=0.009)$, and their ASA score was higher $(+0.3 ; \mathrm{p}=0.01)$. In terms of indications, patients with megacolon or perforation were more likely to undergo an open procedure.

\section{Perioperative outcomes}

The perioperative outcomes are summarized in Table 2. Mean operative time was 201.4 minutes (range: $65-410)$. Overall, 17 out of patients (14.5\%) had conversion from a laparoscopic to an open procedure. The most commonly reported causes for conversion were high BMI, technical problems (lack of space, dilated bowel), or bleeding.

Post-operative complications occurred in 58/151 (38.4\%). Most (53.4\%) were minor (Grade I and II) and included: ileus ( $n=8)$, chest infection $(n=3)$, rectal stump leak treated conservatively $(n=3)$, urinary tract infection $(n=3)$, wound infection $(n=2)$, portal or superior mesenteric vein thrombosis $(n=2)$, high stoma output $(n=2)$, blood transfusion $(n=2)$, pulmonary embolism $(n=2)$, upper gastrointestinal bleeding $(n=1)$, bacteremia $(n=1)$, epidural site infection $(n=1)$, and Clostridium difficile infection $(n=1)$. Four patients had Grade IIIa complications: three with 
collections requiring radiological percutaneous drainage, and one patient with acute bleeding requiring radiological embolization. Overall $17 / 58$ patients had Grade IIIb complications and required reoperation: stoma dysfunction $(n=6)$, intra-abdominal collection $(n=3)$, suspicion of intra-abdominal sepsis $(\mathrm{n}=2)$, rectal stump leak $(\mathrm{n}=2)$, peristomal collection $(\mathrm{n}=2)$, wound dehiscence $(\mathrm{n}=1)$, and small bowel obstruction $(\mathrm{n}=1)$. Grade IV complications requiring intensive care occurred in 5/58: intra-abdominal collection $(n=1)$, hypotension $(n=1)$, third degree atrioventricular block $(\mathrm{n}=1)$, infection with Listeria sp. meningitis $(\mathrm{n}=1)$, or bowel ischaemia $(n=1)$.

In the emergency group, preoperative length of stay did not differ in between patients with or without complication ( 3.1 vs. 2.6 days; $\mathrm{p}=0.32$ ).

One patient died (1/151;0.7\%), who was aged 83 and underwent emergency laparoscopic STC. He subsequently developed respiratory distress and was admitted to ITU, where he died on day 7 post-operatively.

Reoperation was necessary in 19/151 (12.6\%) for complications. The median postoperative length of stay was 9 days (range: 3-67). Overall, 17 patients (11.3\%) were readmitted for the following reasons: ileus $(n=3)$, intra-abdominal collections $(n=3)$, suspicion of intra-abdominal sepsis $(n=2)$, stoma dysfunction $(n=4)$, pain $(n=2)$, parastomal abscess $(n=1)$, rectal stump leak $(\mathrm{n}=1)$, and wound dehiscence $(\mathrm{n}=1)$.

When comparing elective and emergency STC, more patients had open STC in the emergency group ( $29 \%$ vs. $9.8 \%$; $=0.007$ ). The operative time was shorter for the emergency STC group (by 26 minutes; $\mathrm{p}=0.02$ ). In terms of morbidity, there were fewer complications in the elective group ( $27.5 \%$ vs. $44 \%$; $\mathrm{p}=0.05)$. Finally, the postoperative length of stay was also shorter in the 
elective group (by 3.6 days; $\mathrm{p}=0.03$ ). Of note, there were no differences in terms of conversion, reoperation rate or readmission.

When comparing the operative approaches, the majority of open cases were performed for an emergency STC. The operative time was shorter for the open group (by 75 minutes; $p=0.001$ ), but with a higher complications rate $(61.88 \%$ vs. $31.6 \%$; $=0.002)$. The postoperative length of stay was shorter after a laparoscopic STC (by 6.9 days; $p=0.0002$ ).

When considering only emergency STC, there were more male patients in the open group (75.9\% vs. $53.5 \%$; $=0.05$ ). However, patient age and ASA score were comparable. Regarding the perioperative outcomes (Table 3), the operative time for laparoscopic emergency STC was longer when compared to open surgery (by 68 minutes; $\mathrm{p}=0.0001$ ). However, there were fewer complications and a shorter postoperative hospital stay in the laparoscopic group. Similar reoperation and readmission rates were observed.

\section{Second stage procedures}

Most patients underwent a second stage procedure (Table 4), with IPAA being the most frequent operation (43\%). Only 4/151 patients had an ileorectal anastomosis (IRA: $2.6 \%$ ) after careful consideration, because of a relative rectal sparing. The mean operative time for the second stage procedure was 173.4 minutes (range: 50-360) with a mean length of stay of 7.8 days (range: 3 28). We observed a complication rate of $18.2 \%$ following a second stage procedure.

When comparing emergency, elective, open, or laparoscopic STC, there were no differences in terms of operative time, postoperative complications or length of stay. However, following an open STC, a patient was more likely to undergo a definitive completion proctectomy and 
permanent end ileostomy than after laparoscopic STC. In other words, excluding patients still on the waiting list from the analysis, patients undergoing laparoscopic STC were more likely to undergo a restorative second-stage procedure (IRA and IPAA) than those having open STC (75.3\% vs. $50 \% ; \mathrm{p}=0.03)$.

\section{DISCUSSION}

The place of STC with ileostomy formation for acute severe colitis, which does not respond to medical therapy, is well established ${ }^{15,16}$. In the last decade a trend towards minimally invasive surgery has been observed and this enthusiasm extends into the field of inflammatory bowel disease ${ }^{5,6}$. We present herein a large cohort study from a single tertiary center, analyzing the approaches and outcomes of STC for UC over 8 years. Our overall results are in accordance with the current literature, notably an acceptable morbidity rate of $38.4 \%$ and an overall mortality rate less than $1 \%$ 1, 7, 17-22. Different large population-based cohort studies recently confirmed these findings with a mortality rate ranging from 0.9 to $5.2 \%$ after STC for $\mathrm{UC}^{23,24}$.

As with other groups, we have noticed a change in operative approach over time, trending from open to laparoscopy ${ }^{8,10}$. In the literature, more than half of STC are still performed by an open approach $^{9}, 10,18,25$; these findings were confirmed in large American databases ${ }^{8,}{ }^{26}$. Overall, $77.5 \%$ of our cases were attempted laparoscopically, and this rate increased to more than $90 \%$ when considering only elective cases. Since 2014, we have performed all STC operations initially by a minimally invasive approach, with favorable outcomes and respectable conversion rate.

Whilst better short-term outcomes can be expected after a minimally invasive approach, as has been shown for colorectal cancer surgery ${ }^{27}$, for UC, data are more mixed ${ }^{9,}{ }^{18,25}$. In our overall 
experience, a laparoscopic approach was associated with lower morbidity (especially for minor complications) and a shorter hospital stay. However, this was at the expense of a longer mean operative time as noted similarly by others ${ }^{8-10,18,25,28-31}$. In fact, both studied populations (open and laparoscopic) were not entirely similar (more male, older, and higher ASA score in the open group), and these discrepancies might at least in part explain the differences in outcomes ${ }^{9}$. However, other groups have reported similar findings with a clear trend towards better shortterm outcomes following a laparoscopic $\mathrm{STC}^{8,10,20,28-32}$. Messenger et al ${ }^{10}$ have recently reported their outcomes after 290 urgent open and laparoscopic STC. Whilst their results include those with Crohn's colitis, they showed a reduction in morphine requirement, a reduction of minor complications rate, and a shorter hospital stay in the laparoscopic group. Similar findings are reported elsewhere ${ }^{9,28-31}$ and are in broad agreement with the current study. Confirming these data, a recent meta-analysis showed less surgical site infection (including superficial wound infection and intra-abdominal abscess formation) and a shorter hospital stay after laparoscopic $\mathrm{STC}^{11}$. Indeed, minimally invasive surgery is well established to be a protective factor for surgical site infection ${ }^{33}$. Moreover, when focusing on emergency procedures, laparoscopic STC still seems to confer benefits by reducing the risk of complications and a shorter hospital stay. Again, these advantages come at a price: a longer operative time (an additional hour on average), which might be a problem when the patient is haemodynamically unstable. In these particular cases, an open STC seems judicious, and this might explain why patients with megacolon or perforation were more likely to undergo an open procedure in our experience.

Furthermore, elective STC seems to have better outcomes, notably in terms of complications and length of stay. If elective and emergency populations are not strictly the same (differences in terms of indication and ASA score), it remains that elective STC should be performed whenever 
possible. Whilst some emergencies cannot be delayed (perforation, megacolon...), potential indication such as refractory colitis might be better served by an elective operation.

Interestingly, small bowel obstruction (SBO), stoma dysfunction, and ileus remain common complications after $\mathrm{STC}^{7}, 10,17,18,20,21$, as seen in our series. Alves et $\mathrm{al}^{17}$ found $15 \%$ of patients developed SBO in their large open series, requiring re-laparotomy in $8 \%$ of cases. The use of laparoscopy might potentially reduce this risk, but this remains a predominantly hypothetical assertion. On the other hand, the lack of intraperitoneal adhesions associated with laparoscopy ${ }^{34}$ might increase the risk of ileostomy-related volvulus ${ }^{10,35}$.

The fate and the recommended length of the rectal stump continue to fuel debate $\mathrm{e}^{36-38}$. The risk of rectal stump dehiscence or "blowout" has motivated some groups to advocate the use of a rectosigmoid mucous fistula ${ }^{17,19}$. In our reported experience, five patients (3.3\%) presented with this complication, of which only two required reoperation. Our data are in accordance with previous studies $8,9,19,36$, which demonstrated the safety of an intra-peritoneal stump divided at the level of rectosigmoid junction. This strategy does not seem to preclude a further restorative procedure. Routine use of a postoperative rectal catheter might be advantageous in this situation $^{10,16,36,39}$, as a cheap and relatively effective way to reduce rectal stump leak.

STC is usually the first surgical step for the treatment of refractory UC. In accordance with others $^{40}$, we have shown that up to one third of STC are performed electively as a three-stage procedure. Desire for childbearing, medications (steroids, anti-TNF), and poor nutritional status are just a few of the potential explanations for this approach. Second-stage options are usually 
IPAA, completion proctectomy, or ileorectal anastomosis. The latter has been suggested in patients with relative rectal-sparing $\mathrm{UC}^{410}$. In contrast to other centers ${ }^{17}{ }^{410}$, ileorectal anastomosis remains unusual in our practice. Two thirds of our patients underwent a second stage procedure, mainly IPAA and completion proctectomy. Interestingly, more patients undergoing laparoscopic STC had a restorative procedure with IPAA or IRA in comparison to open STC. Again, some of the patients' features in the open group (a slightly older predominantly male population) might partly account for this choice. The decision to proceed with IPAA (and IRA) or completion proctectomy with end ileostomy is based on an in-depth discussion between the surgeon and the patient. Depending on patient expectations, both procedures are reasonable options. However, it is not clear if an open STC might influence the surgeon to preferentially suggest completion proctectomy and this remains speculative. In addition, like others ${ }^{40}$, one third of our overall population is under active endoscopic surveillance awaiting a second-stage procedure; these results should therefore be interpreted with caution. In the literature, the type of second-stage procedure does not seem to be related to the initial approach $^{10,32}$.

Whilst contributing new information regarding the role of STC for UC, this study has several limitations that deserve comment. First, by its retrospective nature, no randomization was performed between groups. This is an obvious bias. In addition, a heterogeneous group of patients with UC has been presented and inevitable differences exist between the groups of patients. Considering the complexity of the minimally invasive approach, a selection bias among patients suitable for laparoscopic versus open surgery may arise, with open surgery preferentially 
offered to patients who have anticipated technical difficulties or higher surgical risks ${ }^{9}$. That said, this series shows that laparoscopic STC is feasible but safe, even in the emergency situation.

\section{CONCLUSIONS}

We present herein a large cohort study from a single teaching institution, analyzing the outcomes following STC for UC over 8 years. We have shown that laparoscopic STC for UC is feasible and safe, even in an emergency situation. Whilst patient differences existed between the compared groups, the laparoscopic approach seems to offer the advantages of notably less morbidity and a shorter hospital stay, at the expense of a longer operative time.

\section{REFERENCES}

1. Bewtra M, Newcomb CW, Wu Q, et al. Mortality Associated With Medical Therapy Versus Elective Colectomy in Ulcerative Colitis: A Cohort Study. Annals of internal medicine 2015; 163(4): 262-70.

2. Ho GT, Mowat C, Goddard CJ, et al. Predicting the outcome of severe ulcerative colitis: development of a novel risk score to aid early selection of patients for second-line medical therapy or surgery. Alimentary pharmacology \& therapeutics 2004; 19(10): 1079-87.

3. Burisch J, Jess T, Martinato M, Lakatos PL, EpiCom E. The burden of inflammatory bowel disease in Europe. Journal of Crohn's \& colitis 2013; 7(4): 322-37.

4. Dinesen LC, Walsh AJ, Protic MN, et al. The pattern and outcome of acute severe colitis. Journal of Crohn's \& colitis 2010; 4(4): 431-7.

5. Seifarth C, Ritz JP, Kroesen A, Buhr HJ, Groene J. Effects of minimizing access trauma in laparoscopic colectomy in patients with IBD. Surgical endoscopy 2015; 29(6): 1413-8. 
6. Tan JJ, Tjandra JJ. Laparoscopic surgery for ulcerative colitis - a meta-analysis. Colorectal disease : the official journal of the Association of Coloproctology of Great Britain and Ireland 2006; 8(8): 626-36.

7. Gu J, Stocchi L, Remzi F, Kiran RP. Factors associated with postoperative morbidity, reoperation and readmission rates after laparoscopic total abdominal colectomy for ulcerative colitis. Colorectal disease : the official journal of the Association of Coloproctology of Great Britain and Ireland 2013; 15(9): 1123-9.

8. Causey MW, Stoddard D, Johnson EK, et al. Laparoscopy impacts outcomes favorably following colectomy for ulcerative colitis: a critical analysis of the ACS-NSQIP database. Surgical endoscopy 2013; 27(2): 603-9.

9. Gu J, Stocchi L, Remzi FH, Kiran RP. Total abdominal colectomy for severe ulcerative colitis: does the laparoscopic approach really have benefit? Surgical endoscopy 2014; 28(2): $617-25$.

10. Messenger DE, Mihailovic D, MacRae HM, O'Connor BI, Victor JC, McLeod RS. Subtotal colectomy in severe ulcerative and Crohn's colitis: what benefit does the laparoscopic approach confer? Diseases of the colon and rectum 2014; 57(12): 1349-57.

11. Bartels SA, Gardenbroek TJ, Ubbink DT, Buskens CJ, Tanis PJ, Bemelman WA. Systematic review and meta-analysis of laparoscopic versus open colectomy with end ileostomy for non-toxic colitis. Br J Surg 2013; 100(6): 726-33.

12. Gu J, Stocchi L, Geisler DP, Kiran RP. Staged restorative proctocolectomy: laparoscopic or open completion proctectomy after laparoscopic subtotal colectomy? Surgical endoscopy 2011; 25(10): 3294-9. 
13. Truelove SC, Witts LJ. Cortisone in ulcerative colitis; final report on a therapeutic trial. British medical journal 1955; 2(4947): 1041-8.

14. Dindo D, Demartines N, Clavien PA. Classification of surgical complications: a new proposal with evaluation in a cohort of 6336 patients and results of a survey. Ann Surg 2004;

240(2): 205-13.

15. Mowat C, Cole A, Windsor A, et al. Guidelines for the management of inflammatory bowel disease in adults. Gut 2011; 60(5): 571-607.

16. Brown SR, Haboubi N, Hampton J, George B, Travis SP, Acpgbi. The management of acute severe colitis: ACPGBI position statement. Colorectal disease : the official journal of the Association of Coloproctology of Great Britain and Ireland 2008; 10 Suppl 3: 8-29.

17. Alves A, Panis Y, Bouhnik Y, Maylin V, Lavergne-Slove A, Valleur P. Subtotal colectomy for severe acute colitis: a 20-year experience of a tertiary care center with an aggressive and early surgical policy. Journal of the American College of Surgeons 2003; 197(3): $379-85$.

18. Coakley BA, Telem D, Nguyen S, Dallas K, Divino CM. Prolonged preoperative hospitalization correlates with worse outcomes after colectomy for acute fulminant ulcerative colitis. Surgery 2013; 153(2): 242-8.

19. Gu J, Stocchi L, Remzi F, Kiran RP. Intraperitoneal or subcutaneous: does location of the (colo)rectal stump influence outcomes after laparoscopic total abdominal colectomy for ulcerative colitis? Diseases of the colon and rectum 2013; 56(5): 615-21.

20. Marceau C, Alves A, Ouaissi M, Bouhnik Y, Valleur P, Panis Y. Laparoscopic subtotal colectomy for acute or severe colitis complicating inflammatory bowel disease: a case-matched study in 88 patients. Surgery 2007; 141(5): 640-4. 
21. Randall J, Singh B, Warren BF, Travis SP, Mortensen NJ, George BD. Delayed surgery for acute severe colitis is associated with increased risk of postoperative complications. $\mathrm{Br} J$ Surg 2010; 97(3): 404-9.

22. Teeuwen PH, Stommel MW, Bremers AJ, van der Wilt GJ, de Jong DJ, Bleichrodt RP. Colectomy in patients with acute colitis: a systematic review. J Gastrointest Surg 2009; 13(4): 676-86.

23. Nordenvall C, Ekbom A, Bottai M, Smedby KE, Nilsson PJ. Mortality after total colectomy in 3084 patients with inflammatory bowel disease: a population-based cohort study. Alimentary pharmacology \& therapeutics 2014; 40(3): 280-7.

24. Tottrup A, Erichsen R, Svaerke C, Laurberg S, Srensen HT. Thirty-day mortality after elective and emergency total colectomy in Danish patients with inflammatory bowel disease: a population-based nationwide cohort study. BMJ Open 2012; 2(2): e000823.

25. Parnaby CN, Ramsay G, Macleod CS, Hope NR, Jansen JO, McAdam TK.

Complications after laparoscopic and open subtotal colectomy for inflammatory colitis: a casematched comparison. Colorectal disease : the official journal of the Association of Coloproctology of Great Britain and Ireland 2013; 15(11): 1399-405.

26. Greenstein AJ, Romanoff AM, Moskowitz AJ, Sosunov EA, Khaitov S, Egorova NN. Payer status and access to laparoscopic subtotal colectomy for ulcerative colitis. Diseases of the colon and rectum 2013; 56(9): 1062-7.

27. van der Pas MH, Haglind E, Cuesta MA, et al. Laparoscopic versus open surgery for rectal cancer (COLOR II): short-term outcomes of a randomised, phase 3 trial. Lancet Oncol 2013; 14(3): 210-8. 
28. Dunker MS, Bemelman WA, Slors JF, van Hogezand RA, Ringers J, Gouma DJ.

Laparoscopic-assisted vs open colectomy for severe acute colitis in patients with inflammatory bowel disease (IBD): a retrospective study in 42 patients. Surgical endoscopy 2000; 14(10): 9114.

29. Seshadri PA, Poulin EC, Schlachta CM, Cadeddu MO, Mamazza J. Does a laparoscopic approach to total abdominal colectomy and proctocolectomy offer advantages? Surgical endoscopy $2001 ; 15(8): 837-42$.

30. Marcello PW, Milsom JW, Wong SK, Brady K, Goormastic M, Fazio VW. Laparoscopic total colectomy for acute colitis: a case-control study. Diseases of the colon and rectum 2001; 44(10): 1441-5.

31. Telem DA, Vine AJ, Swain G, et al. Laparoscopic subtotal colectomy for medically refractory ulcerative colitis: the time has come. Surgical endoscopy 2010; 24(7): 1616-20. 32. Chung TP, Fleshman JW, Birnbaum EH, et al. Laparoscopic vs. open total abdominal colectomy for severe colitis: impact on recovery and subsequent completion restorative proctectomy. Diseases of the colon and rectum 2009; 52(1): 4-10.

33. Gervaz P, Bandiera-Clerc C, Buchs NC, et al. Scoring system to predict the risk of surgical-site infection after colorectal resection. Br J Surg 2012; 99(4): 589-95.

34. Bartels SA, Vlug MS, Henneman D, Ponsioen CY, Tanis PJ, Bemelman WA. Less adhesiolysis and hernia repair during completion proctocolectomy after laparoscopic emergency colectomy for ulcerative colitis. Surgical endoscopy 2012; 26(2): 368-73.

35. Nieto T, Shaikh I, Clark J, Farrands P. Twisting of distal ileum around end ileostomy: is it a specific complication after laparoscopic subtotal colectomy? J Laparoendosc Adv Surg Tech A 2011; 21(2): 161-3. 
36. Brady RR, Collie MH, Ho GT, Bartolo DC, Wilson RG, Dunlop MG. Outcomes of the rectal remnant following colectomy for ulcerative colitis. Colorectal disease : the official journal of the Association of Coloproctology of Great Britain and Ireland 2008; 10(2): 144-50.

37. Longo WE. The out-of-circuit rectum in ulcerative colitis: the bumpy road less traveled: Comment on " Fate of rectal stump after subtotal colectomy for ulcerative colitis in the era of ileal pouch-anal anastomosis". JAMA surgery 2013; 148(5): 412.

38. Buchs NC, Mortensen NJ, Guy RJ, George BD. Persistent colitis after emergency laparoscopic subtotal colectomy for ulerative colitis: a cautionary note. Colorectal disease : the official journal of the Association of Coloproctology of Great Britain and Ireland 2015: in press. 39. Karch LA, Bauer JJ, Gorfine SR, Gelernt IM. Subtotal colectomy with Hartmann's pouch for inflammatory bowel disease. Diseases of the colon and rectum 1995; 38(6): 635-9.

40. Munie S, Hyman N, Osler T. Fate of the rectal stump after subtotal colectomy for ulcerative colitis in the era of ileal pouch-anal anastomosis. JAMA surgery 2013; 148(5): 408-11. 41. Myrelid P, Oresland T. A reappraisal of the ileo-rectal anastomosis in ulcerative colitis. Journal of Crohn's \& colitis 2015; 9(6): 433-8. 